\title{
Measuring Social Value Orientation
}

\author{
Ryan O. Murphy* Kurt A. Ackermann ${ }^{\dagger} \quad$ Michel J. J. Handgraaf ${ }^{\ddagger}$
}

\begin{abstract}
Narrow self-interest is often used as a simplifying assumption when studying people making decisions in social contexts. Nonetheless, people exhibit a wide range of different motivations when choosing unilaterally among interdependent outcomes. Measuring the magnitude of the concern people have for others, sometimes called Social Value Orientation (SVO), has been an interest of many social scientists for decades and several different measurement methods have been developed so far. Here we introduce a new measure of SVO that has several advantages over existent methods. A detailed description of the new measurement method is presented, along with norming data that provide evidence of its solid psychometric properties. We conclude with a brief discussion of the research streams that would benefit from a more sensitive and higher resolution measure of SVO, and extend an invitation to others to use this new measure which is freely available.
\end{abstract}

Keywords: Social Value Orientation (SVO), social preferences, narrow self-interest, measurement methods, individual differences.

\section{Introduction}

The assumption of narrow self-interest is central to rational choice theory. The postulate is that decision makers (DMs) are concerned about maximizing their own material gain, indifferent to the payoffs of other DMs around them. This is a simplifying assumption that yields a powerful framework to predict and explain human decision making behavior across a wide variety of domains. However there are reliable counterexamples demonstrating that DMs' elicited preferences and choices are often influenced in part by the payoffs of other DMs, thus challenging what some have termed the selfishness axiom (Henrich et al., 2005).

Studies on the motivations that underlie interdependent decision behavior have a long history and these motivations have been referred to by a variety of names, including: social preferences, social motives, other-regarding preferences, welfare tradeoff ratios, and Social Value Orientation (SVO). For consistency, we refer to this construct as SVO for the remainder of this paper. Within the SVO framework it is assumed that people vary in their motivations or goals when evaluating different re-

This research has been supported in part by United States National Science Foundation grant SES-0637151. Additional thanks to Mike Kuhlman, Jeff Joireman, Michael Schulte-Mecklenbeck, and Miguel Fonseca for their constructive feedback on early versions of this paper.

${ }^{*}$ ETH Zürich, Chair of Decision Theory and Behavioral Game Theory, Clausiusstrasse 50, 8092 Zürich, Switzerland. Email: rmurphy@ethz.ch.

${ }^{\dagger}$ ETH Zürich, Chair of Decision Theory and Behavioral Game Theory, Clausiusstrasse 50, 8092 Zürich, Switzerland.

¥Wageningen University, Economics of Consumers and Households (ECH), Hollandseweg 1, 6706 KN, Wageningen, The Netherlands. source allocations between themselves and another person. As examples, a DM may endeavor to maximize her own payoff (individualistic), maximize (competitive) or minimize (inequality averse) the difference between her own and the other person's payoff, or maximize joint payoffs (prosocial). It is worth noting, however, that the assumption of narrow self-interest is itself a particular SVO, namely a perfectly individualistic orientation. Moreover considering a spectrum of different SVOs is not a challenge to rational choice theory per se, but rather the extension of a postulate in an effort to increase the theory's psychological realism and descriptive accuracy.

SVO has been found to affect cognitions and account for behavior across a range of interpersonal decision making contexts, specifically in the domain of negotiation settings (De Dreu \& Boles, 1998) and resource dilemmas (Roch et al., 2000; Roch \& Samuelson, 1997; Samuelson, 1993). SVO has also been identified as a covariate, interacting with different emotional states and influencing the propensity to cooperate (Zeelenberg, Nelissen, Breugelmans \& Pieters, 2008). SVOs have even been identified in non-human primates (Burkart, Fehr, Efferson \& van Schaik, 2007), indicating that some other species also show intrinsic preferences for prosocial behavior.

In order to use the full explanatory power of SVO as a psychological construct, we need to measure it efficiently, reliably and validly. Several different measurement methods for quantifying variations in SVO across individuals have been developed (for overviews, see McClintock \& Van Avermaet, 1982; Au \& Kwong, 2004; Murphy \& Ackermann, 2011). Although the use of existent measures has produced a wealth of findings even 
with categorical approaches (see, for instance, De Dreu \& Boles, 1998; Kuhlman \& Marshello, 1975a, 1975b; Van Lange \& Visser, 1999), these measures have substantial limitations. For instance, some measures yield only low-resolution output that lack sensitivity to important individual differences, providing at best a nominal categorization (e.g., the Triple-Dominance Measure, see Van Lange, Otten, De Bruin \& Joireman, 1997). Other measures are highly inefficient and often fail to produce consistent results for a substantial proportion of subjects (e.g., the Ring Measure, see Liebrand, 1984). Yet other methods require substantial time and effort from a research subject in order to produce a score (e.g., Utility and Conjoint Measurement procedures, or Regression and Clustering techniques, see Wyer, 1969; Radzicki, 1976; or Knight \& Dubro, 1984, respectively). Moreover, none of these existent measures are explicitly designed to detect more nuanced motivations like inequality aversion. Specifically, previous measures have not disentangled the orientation of joint gain maximization from the motivation to minimize the difference between outcomes. Although these two orientations are related in that they both indicate a deviation from individualism towards prosociality, they are substantially different motivations, which should be differentiated both theoretically and operationally.

Furthermore, Social Value Orientation is a continuous construct, as it corresponds to the quantity of how much a DM is willing to sacrifice in order to make another DM better off (or perhaps worse off). This quantification of interdependent utilities can be best represented on a continuous scale. Moreover, since the most commonly used SVO measures to date produce only categorical data, a substantial amount of information related to peoples' social preferences is being discarded and ignored. Consequently, the full explanatory power of SVO has not been used because of this unnecessary sacrifice of statistical power (see Cohen [1983] for a discussion of the unfortunate practice of reducing continuous variables to categories).

In our view, a method for assessing SVO should yield high resolution output which makes it sensitive to interand intra-individual differences and facilitate comparisons thereof, be easy to use, be efficient, be able to detect the most prevalent SVO individual differences, allow for an evaluation of rank orders of social preferences, and yield meaningful results for virtually all subjects. Amongst these criteria, we consider the demand for a high resolution measure which produces data on a continuous scale as crucial.

We introduce here a new measure of SVO, which takes this conceptualization into account and allows for greater explanatory potential of SVO through increased statistical power while also meeting the aforementioned psycho-
Figure 1: This shows the six primary SVO Slider items as seen by the subjects.

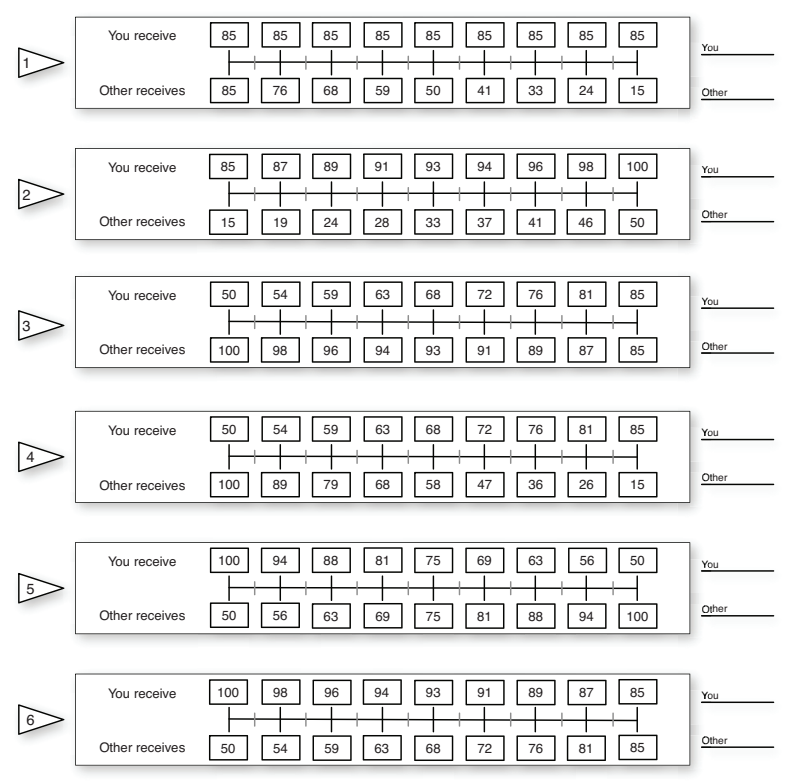

metric criteria. This new method is referred to as the $S V O$ Slider Measure. We provide a detailed discussion of this new measure, along with norming data and evidence of the new measure's strong psychometric properties.

\section{The SVO Slider Measure}

The SVO Slider Measure can be administered as a paper based choice task or as an online measure. The measure has six primary items with nine secondary (and optional) items. All of the items have the same general form. Each item is a resource allocation choice over a well defined continuum of joint payoffs. For example, consider a DM choosing a value $x$ between 50 and 100 inclusive. Her payoff would be $x$, whereas the other's payoff would be $150-x$. The DM would indicate her allocation choice by marking a line at the point that defines her most preferred joint distribution (see item 5 in Figure 1, see also Table 7, p. 779). After the DM has marked her most preferred allocation, she would write the corresponding payoffs resulting from her choice to the right of the item. Although this step of writing the values is redundant, it serves to verify that the DM understood the choice task and the resulting allocations.

\subsection{Primary SVO slider items}

The six primary Slider Measure items are shown in Figure 1. These six items were derived from the six lines 
Figure 2: This figure shows where in the self/other allocation plane the six primary items are from the Slider Measure.

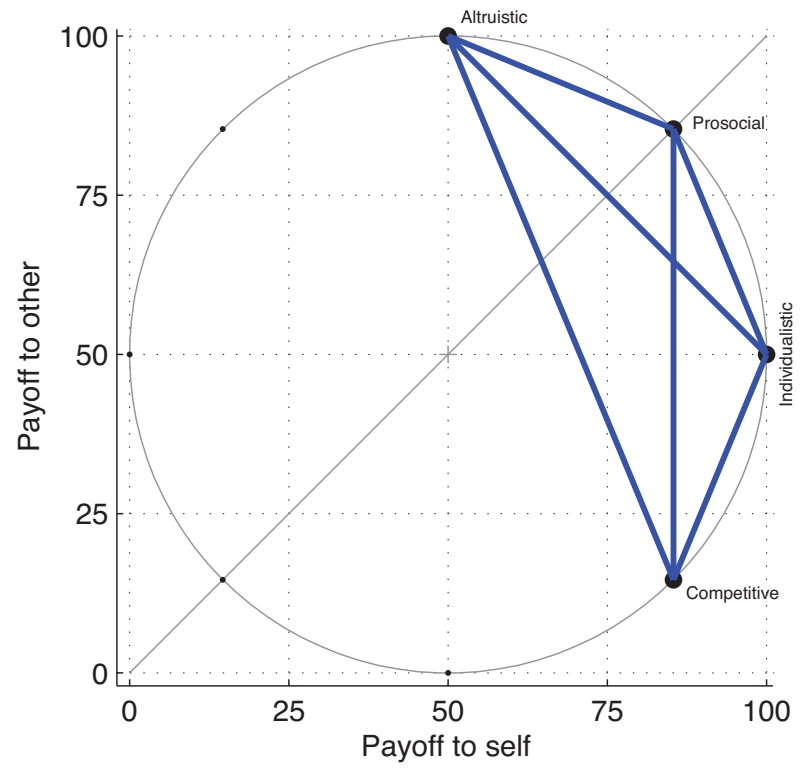

that fully interconnect the four points corresponding to the most common idealized social orientations reported in the literature (altruistic, prosocial, individualistic, and competitive; see Figure 2). A DM evaluates each of the items sequentially and for each one indicates her most preferred joint distribution. The set of responses can then be scored to yield a single score for the DM, the rank order of her social preferences, and additionally contains a check for transitivity in her revealed preferences.

The SVO Slider Measure has several advantages. First, the responses can be evaluated for comprehension (e.g., checking the correspondence between the mark on the distribution line and the written distribution values). Second, the responses can be evaluated for transitivity. Although SVO is a matter of subjective preferences, these preferences should conform to the elemental requirement of transitivity. Random responding would likely result in an intransitive set of responses. Third, the responses yield a full ranking of preferences over motivations. Fourth, the measure can be scored in a straight-forward manner to yield a single index of SVO as follows. The mean allocation for self $\left(\bar{A}_{s}\right)$ is computed as is the mean allocation for the other $\left(\bar{A}_{o}\right)$. Then 50 is subtracted from each of these means in order to "shift" the base of the resulting angle to the center of the circle $(50,50)$ rather than having its base start at the Cartesian origin. Finally, the inverse tangent of the ratio between these means is computed, resulting in a single index of a person's SVO.

$$
\mathrm{SVO}^{\circ}=\arctan \left(\frac{\left(\bar{A}_{o}-50\right)}{\left(\bar{A}_{s}-50\right)}\right)
$$

This response format is highly sensitive to individual differences and yields an individual score at the ratio level of measurement. Assessing SVO in this way also facilitates parameterization and model assessment that is not possible with other existent measures. Nonetheless, reducing the high resolution score to a nominal category may be desirable in some cases (e.g., to compare new results to previous studies), and the resulting SVO Slider angles can be transformed into corresponding categories with ease as follows.

If a person would choose the option that maximizes the allocation for the other in each of the six primary items, the resulting angle would be $61.39^{\circ}$, indicating perfect altruism. A prosocial DM with inequality aversion would yield an angle of $37.48^{\circ}$. A prosocial DM who endeavored to maximize joint gain (and is inequality tolerant) would yield an angle between $37.09^{\circ}$ and $52.91^{\circ}$. The reason for this range is that this DM would be wholly indifferent across the entire SVO Slider item that has a slope of -1 (i.e., the item with endpoints 100, 50 and $50,100)$ as it has a constant sum. A perfectly consistent individualist yields an angle between $-7.82^{\circ}$ and $7.82^{\circ}$. The reason for this range is that this particular DM would be wholly indifferent across the range of outcomes contained in the SVO Slider item that has an undefined slope (endpoints 85,85 and 85,15 ). A perfectly consistent competitor yields an angle of $-16.26^{\circ}$.

Given the angles that result from idealized SVO types, proper boundaries between categories can be derived by bisecting the respective adjacent ranges. Altruists would have an angle greater than $57.15^{\circ}$; prosocials would have angles between $22.45^{\circ}$ and $57.15^{\circ}$; individualists would have angles between $-12.04^{\circ}$ and $22.45^{\circ}$; and competitive types would have an angle less than $-12.04^{\circ}$. As it can be seen, these boundaries are not at intuitive locations. The reason for this is that the Slider Measure only uses a subset of possible items from the allocation plane and these items are not symmetrically distributed around the whole of the ring. Because only an asymmetric set of items is used here, the resulting convex hull of possible scores is "squished" to the upper-right, relative to the midpoint of the ring. This characteristic does not adversely affect the validity of the measure.

\subsection{Secondary SVO slider items}

There are nine secondary SVO Slider Measure items. This set of items is explicitly designed to disentangle the prosocial motivations of joint maximization from inequality aversion. The items are defined in the prosocial area of the self/other allocation plane and have approximately the same magnitude (ranging between 50 and 100 value units) as the six primary items. One noteworthy feature of these secondary items is that all of the distribution 
ranges intersect the diagonal line. This is an important feature of the set as points on the diagonal line correspond to perfectly equal allocations, i.e., those distributions that minimize inequality between the DM and the other person. A person motivated to minimize inequality would make allocations on or very near the $45^{\circ}$ line. Conversely a person motivated to maximize joint gains would make allocations at the endpoints, as far from the diagonal as possible as it turns out, as these allocations maximize collective earnings. Previous measures of SVO have not been explicitly designed to make a differentiation between these two motivations. The nine items are shown in Figures 6 and 7. An example of results from these items is discussed in Section \$3.6.

\subsection{Web-based SVO slider measure}

In addition to being administrable as a paper-based measure, the Slider Measure has been programmed as an online research tool which can be freely used by any researcher. ${ }^{1}$ The online measure and supporting material, as well as the paper based versions of the new measure, can be found at: http://vlab.ethz.ch/svo/SVO_Slider/.

With the online SVO Slider Measure, items are presented in a random order. Subjects record their choices by moving a webpage slider input back and forth, changing the joint allocations until they find their most preferred joint outcome (see Figure 3 for a screen shot). The online items are dynamic and display information is updated in real time as the DM moves the slider over the option space. The choice procedure is the same for all of the items. After the subjects have participated, the researcher is sent an email with the datafile attached; the datafile contains the subjects' identifying information, date/time stamp, item order, and all of the DMs' allocation choices.

\section{Psychometric properties of the SVO Slider Measure}

\subsection{Slider Measure validation procedure}

In order to assess the psychometric properties of the new SVO Slider Measure it was tested in tandem with the established and most commonly used measures of SVO;

\footnotetext{
${ }^{1}$ Computing results from the SVO Slider (checking for transitivity, establishing the ranking of preferences, and finding a subject's SVO angle) can be somewhat demanding and thus we have developed an analysis script that automates and simplifies this process. This script is available for download from the SVO website along with a detailed tutorial on its use. We also provide an Excel worksheet for researchers who are interested in quick and basic results.
}

Figure 3: Online Slider Measure.

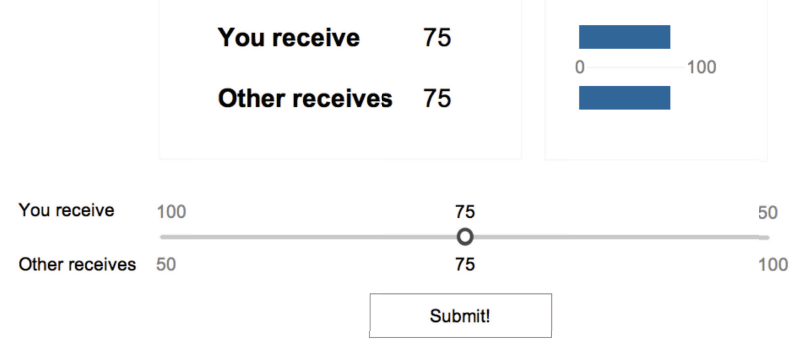

namely the Triple-Dominance Measure (see Van Lange et al., 1997) and the Ring Measure (Liebrand, 1984). Fifty-six individuals from various majors were recruited to participate in a multi-part "decision making study" at a European university. No deception was used in this research and subjects were guaranteed strict confidentiality for all of their choices. The choices in the experiment were made incentive compatible by means of a lottery-for each experimental session four subjects were randomly selected after making their choices and for each selected person one of their allocation decisions was implemented (i.e., their allocation choice was carried out such that they received some chosen payoff, as well as did some other randomly selected person, according to their actual choice). For all research sessions, subjects were reminded that their decisions were private and that there was a real chance that their choices would have a pecuniary effect upon themselves and some other person if they happened to be selected by lottery. DMs selected by lottery were paid privately in cash within a week of their participation. Each unit of value in the experiment corresponded to 50 Swiss cents and the average earnings were 81.70 Swiss francs (US\$77) per paid subject.

Three research sessions were run, with one week separating the sessions. Each research session required fewer than 15 minutes to conduct and used paper based methods. In the first session, subjects completed the 9-item Triple-Dominance Measure and the 24-item Ring Measure. In the second session, subjects completed the Slider Measure and the Triple-Dominance Measure. In the third session, subjects completed the Ring Measure and the Slider Measure. All of the measures used standardized values between 0 and 100 . This research design allowed us to assess the test-retest reliability of the TripleDominance Measure, the Ring Measure, and the Slider Measure. It also allowed us to compute the associations between the different measures and establish norming data and convergent validity for the new SVO Slider Measure. 
Table 1: The percentage of individuals that were assigned to each of the different SVO categories by the different measurement methods (TD- Triple Dominance, RMRing Measure, SM- Slider Measure), ordered by experimental session.

\begin{tabular}{lccccccc}
\hline & Session 1 & Session 2 & Session 3 & \\
& & & & & & & Grand \\
& TD & RM & TD & SM & RM & SM & mean \\
\hline Prosocial & 59 & 53 & 61 & 58 & 58 & 64 & 59 \\
Individualistic & 21 & 45 & 32 & 39 & 36 & 34 & 35 \\
Competitive & 2 & 2 & 3 & 3 & 4 & 2 & 3 \\
Unclassifiable & 18 & 0 & 3 & 0 & 2 & 0 & 4 \\
\hline
\end{tabular}

Table 2: A cross tabulation showing the frequency of categorization from test-retest between session 1 (S 1) and session 2 (S 2) for the Triple-Dominance Measure.

\begin{tabular}{|c|c|c|c|c|c|}
\hline & & \multicolumn{4}{|c|}{ S 2} \\
\hline & & Prosocial & Individualistic & Competitive & Unclassifiable \\
\hline \multirow{4}{*}{ S 1} & Prosocial & 23 & 1 & 0 & 1 \\
\hline & Individualistic & 2 & 8 & 0 & 0 \\
\hline & Competitive & 0 & 1 & 0 & 0 \\
\hline & Unclassifiable & 3 & 5 & 1 & 1 \\
\hline
\end{tabular}

\subsection{Results}

Table 1 shows the percentage of individuals that were assigned to each of the different SVO categories by the different measurement methods, ordered by experimental session. Across all measurement methods there is a clear majority type, namely prosocial, occurring about $59 \%$ of the time. Individualist is less common, but found about $35 \%$ of the time. Competitive and unclassifiable types complete the remainder of the sample representing about $3-4 \%$ each.

\subsection{Reliability}

\subsubsection{Triple-Dominance Measure test-retest reliabil- ity}

Forty-six subjects completed both sessions 1 and 2. Of those, $32(23+8+0+1)$ were categorized in the same SVO category each time by the Triple-Dominance Measure, yielding a consistency of $70 \%$ (Goodman and Kruskal's gamma. ${ }^{2}=0.391$ )

\footnotetext{
${ }^{2}$ As the Triple-Dominance Measure yields a nominal level variable with more than two categories, a Pearson product moment correlation coefficient is not an appropriate statistic for assessing its reliability, hence the non-parametric Gamma statistic is used as an index of testretest association.
}

Table 3: A cross tabulation showing the frequency of categorization from test-retest between session 1 (S 1) and session 3 (S 3) for the Ring Measure.

\begin{tabular}{llcccc}
\hline & & \multicolumn{3}{c}{ S 3 } & \\
& & Prosocial & Individualistic & Competitive & Unclassifiable \\
\hline \multirow{3}{*}{ S 1 } & Prosocial & 18 & 3 & 0 & 0 \\
\cline { 3 - 5 } & Individualistic & 8 & 12 & 1 & 0 \\
\cline { 3 - 5 } & Competitive & 0 & 1 & 0 & 0 \\
Unclassifiable & 0 & 0 & 0 & 0 \\
\hline
\end{tabular}

Table 4: A cross tabulation showing the frequency of categorization from test-retest between session 2 (S 2) and session 3 (S 3) for the primary SVO Slider items.

\begin{tabular}{|c|c|c|c|c|c|}
\hline & \multicolumn{4}{|c|}{ S 3} \\
\hline & & Prosocial & Individualistic & Competitive & Unclassifiable \\
\hline \multirow{4}{*}{ S 2} & Prosocial & 25 & 1 & 0 & 0 \\
\hline & Individualistic & 3 & 15 & 0 & 0 \\
\hline & Competitive & 0 & 1 & 1 & 0 \\
\hline & Unclassifiable & 0 & 0 & 0 & 0 \\
\hline
\end{tabular}

\subsubsection{Ring Measure test-retest reliability}

Forty-four subjects completed both sessions 1 and 3. Of those, $30(18+12+0+0)$ were categorized into the same SVO category each time by the Ring Measure, yielding a consistency of $68 \%$. Further the correlation between the resulting angles from the test-retest of the ring measure was $r=0.599 .^{3}$

\subsubsection{Slider Measure test-retest reliability}

Forty-six subjects completed both sessions 2 and 3. Of those, 41 were categorized in the same SVO category each time by the Slider Measure, yielding a consistency of $89 \%$. Further the correlation between the resulting angles from the test-retest SVO Slider Measure was $r=$ 0.915 .

\subsection{Validity}

\subsubsection{Convergent validity: Categorical agreement}

Across research sessions, the Triple-Dominance Measure and the Ring Measure categorized the same subjects into the same SVO category $67 \%$ of the time. The TripleDominance Measure and the Slider Measure categorized the same subjects in the same SVO category $74 \%$ of the time. The Ring Measure and the Slider Measure catego-

\footnotetext{
${ }^{3}$ In order to verify the robustness of these results, non-parametric statistics of association were also conducted in parallel with Pearson's $r$. The non-parametric tests yielded the same pattern of results.
} 
Table 5: The correlation coefficients between the different sessions and methods. These values show both the test-retest reliabilities, as well as the cross method correlations (in gray) which address convergent validity.

\begin{tabular}{lcccc}
\hline & RM-1 & RM-3 & SM-2 & SM-3 \\
\hline RM-1 & 1 & - & - & - \\
RM-3 & 0.599 & 1 & - & - \\
SM-2 & 0.724 & 0.536 & 1 & - \\
SM-3 & 0.680 & 0.641 & 0.915 & 1 \\
\hline
\end{tabular}

rized the same subjects in the same SVO category $75 \%$ of the time.

\subsubsection{Convergent validity: Correlational agreement}

The Ring Measure and Slider Measure both produce continuous results (in the form of angles within the self/other allocation plane), and these results are amenable to computing correlation coefficients across different measures. Table 5 displays these correlation coefficients, showing both the test-retest reliability of the Ring Measure $(r=0.599)$ and Slider Measure $(r=0.915)$, as well as the correlations between SVO angles across the different measurement methods.

The results show that the Slider Measure correlates as well (if not better) with the Ring Measure as the Ring Measure does with itself across retests. This is strong evidence that the methods are measuring the same thing and further it demonstrates that the Slider Measure is more reliable than the Ring Measure (the mean correlation between the different methods is $r=0.649$ whereas the test-retest correlation for the Ring Measure is only $r=0.599)$.

\subsubsection{Predictive validity}

In order to evaluate the Slider Measure's predictive validity, a second study was conducted where different subjects $(N=100)$ first completed the Slider Measure and then played a one-shot anonymous Prisoner's Dilemma game. Identical to the first study, this study used monetary incentives determined by a lottery. We found a moderate and statistically significant point-biserial correlation ( $r=0.239$ ) between the subjects' SVO angles and their choices in the Prisoner's Dilemma, indicating a positive relation between SVO angle and cooperation, as would be expected. These results are consistent in direction and magnitude with other findings from incentive compatible choice tasks in social dilemmas and measures of SVO (Balliet et al., 2009).
Figure 4: The distribution of SVO scores from the Slider Measure as represented by angles. The dark line is a smoothed kernel density estimation.

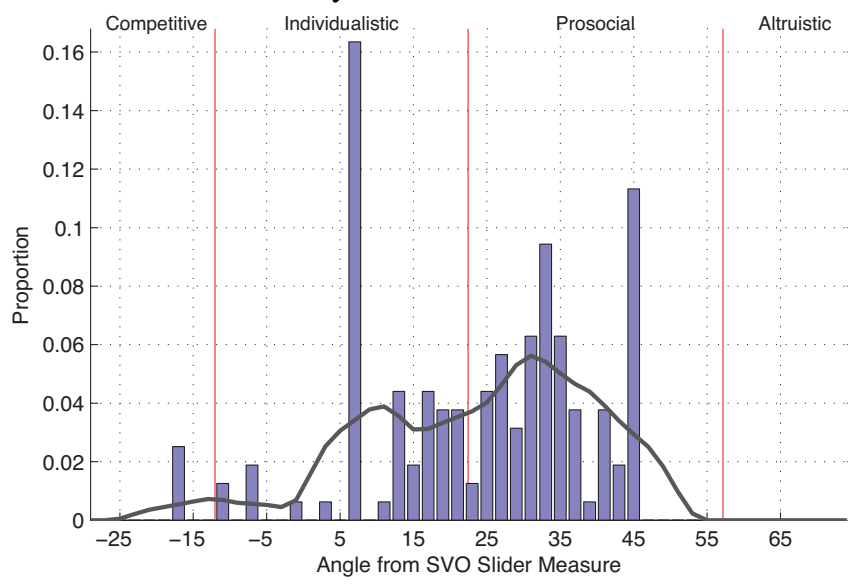

\subsection{Additional results}

As noted before, one advantage of the Slider Measure is its high resolution, as it yields a ratio level of measurement. Previous measures of SVO produce output as a simple categorization, which is a limitation. Conversely, producing a ratio level variable, the distribution of observed SVO angles can be plotted and the density of different orientations can be estimated. Figure 4 shows this distribution. A LOESS (Cleveland \& Devlin, 1988) smoothed kernel density estimation was made of the distribution of SVO scores to provide some general idea of its shape. We find a multimodal distribution of SVO types in our sample. The largest clustering is in the prosocial region shifted slightly to the left (toward individualistic). The second clustering is in the individualistic region and is shifted to the right (toward prosocial). Within this region is the most common SVO score of $7.82^{\circ}$ which corresponds to perfectly individualistic choices. The density function trails off to the left, denoting only a few competitive types. As can be seen in the figure, there is substantial variance in the subjects' SVO angles, beyond what a nominal level categorization would indicate. Moreover, the observed variance supports the assertion that a sensitive SVO measure, which produces reliable high resolution data on a continuous SVO scale, is valuable in that it can capture the rich gradation of social preferences.

As already noted, the transitivity of responses can be assessed with the Slider Measure. We found that $98 \%$ of our subjects produced completely transitive sets of social preference choices. This finding stands in stark contrast to the consistency results from the Ring Measure where only $55 \%$ of the same subjects produced internally consistent results. This would indicate that almost all subjects have well defined social preferences but that the 
Table 6: The full rank orderings of social preferences from the SVO Slider Measure across sessions. Note that $25 \%$ of the decision makers were indifferent between Individualistic and Prosocial allocations when their inferred preferences are reduced to ranks.

\begin{tabular}{llllr}
\hline $\begin{array}{l}\text { First } \\
\text { preference }\end{array}$ & $\begin{array}{l}\text { Second } \\
\text { preference }\end{array}$ & $\begin{array}{l}\text { Third } \\
\text { preference }\end{array}$ & $\begin{array}{l}\text { Least } \\
\text { preferred }\end{array}$ & Percent \\
\hline & & & & \\
Prosocial & Individualistic & Altruistic & Competitive & $27 \%$ \\
Prosocial & Altruistic & Individualistic & Competitive & $25 \%$ \\
Prosocial & Individualistic & Competitive & Altruistic & $13 \%$ \\
(Individualistic & Prosocial) & Competitive & Altruistic & $25 \%$ \\
Individualistic & Competitive & Prosocial & Altruistic & $4 \%$ \\
Individualistic & Prosocial & Altruistic & Competitive & $2 \%$ \\
Competitive & Individualistic & Prosocial & Altruistic & $4 \%$ \\
& & & & \\
\hline
\end{tabular}

Ring Measure is not particularly well suited to measure them.

As an additional feature, the full ranking of people's social preferences can be obtained from evaluating the six primary items of the SVO Slider Measure (see Table 6). These kind of complete ordinal results are not possible with other common SVO measurement methods. Moreover, information about a DM's least preferred allocation may be useful to know when measuring individual differences.

\subsection{Separating the prosocial preferences of inequality aversion and joint maximiza- tion}

The secondary items from the Slider Measure are designed to differentiate between two different prosocial motivations: inequality aversion and joint maximization. As prosocial behavior can arise from both of these underlying motivations, we demonstrate here how to disentangle these motivations using the secondary SVO Slider items.

In order to identify prosocial DM's underlying motivations, two mean difference scores were computed for each prosocial subject from their allocation choices on the secondary Slider Measure items. The first difference score was defined as the average normalized distance between the subject's allocations and the particular allocations that would maximize equality. For example, if a DM always chose allocations that were on the diagonal line (see Figure 7), her mean difference score from idealized inequality aversion would be zero, indicating perfect consistency with the preference of inequality aversion. A second difference score was computed for each subject that was defined as the mean distance between
Figure 5: The distribution of prosocial preferences, ranging from perfect inequality aversion to perfect joint gain maximization. The most common preference is for joint gain maximization $(29 \%)$ but there is substantial variance in DM's prosocial preferences.

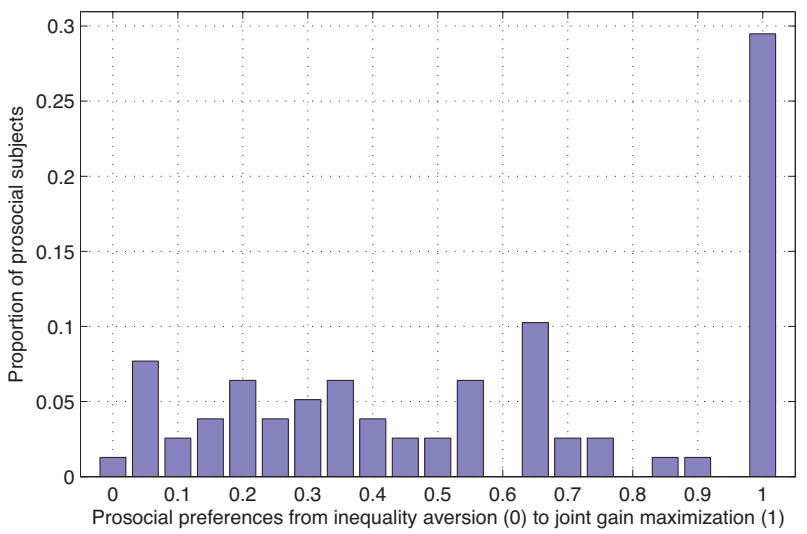

her selected allocations and the particular allocations that maximized joint payoffs for that item. If the mean difference for this second index was zero, it indicates that the DM's allocation choices are perfectly consistent with joint maximization. These values can be meaningfully aggregated into a single index by computing the ratio of the first difference score divided by the sum of both difference scores. The result is an index ranging between 0 (indicating allocation choices perfectly consistent with inequality aversion) and 1 (indicating allocation choices perfectly consistent with a preference for joint gain maximization).

Results were obtained from the 79 DMs who made consistently prosocial allocations in the primary and secondary items across both studies. The distribution of individuals' inequality aversion / joint gain maximization indices is shown in Figure 5. Several results are noteworthy. First, this distribution suggests that prosocial DMs are not homogeneous with respect to their more nuanced prosocial preferences. Some people are striving for maximizing joint gain, whereas others seem to be, at least somewhat, sensitive to equality between payoffs. Second, while the modal preference is for joint gain maximization, a slim majority of DMs are actually closer to inequality aversion. This distribution is both non-uniform and non-skewed with the mean and median at 0.571 . Splitting the sample at $0.5,54 \%$ of DMs would be categorized as inequality averse whereas $45 \%$ would be better described as joint gain maximizers (one person is exactly at the midpoint of 0.5 and is not categorized). Lastly, the shape of the distribution suggests that there is greater conformity in how joint maximizing DMs make allocation choices compared to how inequality averse DMs allocate resources. 


\section{Discussion}

Social preferences are of fundamental importance in understanding interdependent decision making behavior among people. In order to quantify the degree to which people care about outcomes for others, it is necessary to develop reliable measurement methods to assess this construct. Consistent with this goal, we have described a new measure of SVO and demonstrated that it is quick, efficient, easy to implement, has very good psychometric properties, yields scores for individuals at the ratio level, and facilitates comparison to other measures. The advent of a high resolution measure of SVO opens opportunities for different research streams to use social preferences as a dependent variable. These types of studies could address questions regarding how context, information, experience, and framing affect peoples' propensities to make tradeoffs in resources between themselves and others. These lines of research could also answer larger questions like under what conditions is the selfishness axiom a good approximation for explaining human behavior, and when is it insufficient, or even grossly inaccurate. This new measurement method can serve as a bridge between perspectives informed by Homo economicus and those perspectives which take descriptive accuracy as a starting point.

In a broader sense, we would like to encourage scientists interested in human decision making to develop higher resolution measures. Having more sensitive and reliable measurement methods is critical for the detection of subtle yet important effects that may result from changes in context and information. Therefore, we think that the technique employed with the Slider Measure could also be useful in the development of related methods for assessing other individual differences, such as risk perception (e.g., Ganzach, 2000; Ganzach et al., 2008), or temporal discounting (e.g., Stevenson, 1992). In general, we believe that allowing subjects to explore a range of well ordered and intuitive options facilitates not only the revelation of preferences, but also the discovery and unencumbered expression of those preferences.

\section{References}

Au, W. \& Kwong, J. (2004). Measurements and effects of social-value orientation in social dilemmas: A review. In R. Suleiman, D. Budescu, I. Fischer, \& D. Messick, (Eds.), Contemporary Psychological Research on Social Dilemmas, pp. 71-98. New York: Cambridge University Press.

Balliet, D., Parks, C. \& Joireman, J. (2009). Social value orientation and cooperation in social dilemmas: A meta-analysis. Group Processes \& Intergroup Rela- tions, 12, 533-547.

Burkart, J. M., Fehr, E., Efferson, C. \& van Schaik, C. P. (2007). Other-regarding preferences in a non-human primate: Common marmosets provision food altruistically. Proceedings of the National Academy of Sciences, 104, 19762-19766.

Cleveland, W. S. \& Devlin, S. J. (1988). Locally weighted regression: An approach to regression analysis by local fitting. Journal of the American Statistical Association, 83, 596-610.

Cohen, J. (1983). The cost of dichotomization. Applied Psychological Measurement, 7, 249-253.

De Dreu, C. K. W. \& Boles, T. L. (1998). Share and share alike or winner take all?: The influence of social value orientation upon choice and recall of negotiation heuristics. Organizational Behavior and Human Decision Processes, 76, 253-276.

Ganzach, Y. (2000). Judging risk and return of financial assets. Organizational Behavior and Human Decision Processes, 83, 353-370.

Ganzach, Y., Ellis, S., Pazy, A. \& Ricci-Siag, T. (2008). On the perception and operationalization of risk perception. Judgment and Decision Making, 3, 317-324.

Henrich, J., Boyd, R., Bowles, S., Camerer, C., Fehr, E., Gintis, H., McElreath, R., Alvard, M., Barr, A., Ensminger, J., Henrich, N., Hill, K., Gil-White, F., Gurven, M., Marlowe, F., Patton, J. \& Tracer, D. (2005). "Economic man" in cross-cultural perspective: Behavioral experiments in 15 small-scale societies. Behavioral and Brain Sciences, 28, 795-855.

Knight, G. P. \& Dubro, A. F. (1984). Cooperative, competitive, and individualistic social values - an individualized regression and clustering approach. Journal of Personality and Social Psychology, 46, 98-105.

Kuhlman, D. M. \& Marshello, A. F. (1975a). Individual differences in the game motives of own, relative, and joint gain. Journal of Research in Personality, 9, 240251.

Kuhlman, D. M. \& Marshello, A. F. (1975b). Individual differences in game motivation as moderators of preprogrammed strategy effects in prisoner's dilemma. Journal of Personality and Social Psychology, 32, 922931.

Liebrand, W. (1984). The effect of social motives, communication and group-size on behavior in an $n$-person multi-stage mixed-motive game. European Journal of Social Psychology, 14, 239-264.

McClintock, C. \& Van Avermaet, E. (1982). Social values and rules of fairness: A theoretical perspective. In V. Derlega \& J. Grzelak, (Eds.), Cooperation and Helping Behavior, pp. 43-71. New York: Academic Press.

Murphy, R. O. \& Ackermann, K. A. (2011). A review of measurement methods for social preferences. Working 
Paper, Chair of Decision Theory and Behavioral Game Theory, ETH Zürich.

Radzicki, J. (1976). Technique of conjoint measurement of subjective value of own and other's gains. Polish Psychological Bulletin, 7, 179-186.

Roch, S. G., Lane, J. A. S., Samuelson, C. D., Allison, S. T. \& Dent, J. L. (2000). Cognitive load and the equality heuristic: A two-stage model of resource overconsumption in small groups. Organizational Behavior and Human Decision Processes, 83, 185-212.

Roch, S. G. \& Samuelson, C. D. (1997). Effects of environmental uncertainty and social value orientation in resource dilemmas. Organizational Behavior and $\mathrm{Hu}$ man Decision Processes, 70, 221-235.

Samuelson, C. D. (1993). A multiattribute evaluation approach to structural change in resource dilemmas. Organizational Behavior and Human Decision Processes, 55, 298-324.

Stevenson, M. K. (1992). The impact of temporal context and risk on the judged value of future outcomes. Organizational Behavior and Human Decision Processes, 52, 455-491.

Van Lange, P. A. M., Otten, W., De Bruin, E. \& Joireman, J. (1997). Development of prosocial, individualistic, and competitive orientations: Theory and preliminary evidence. Journal of Personality and Social Psychology, 73, 733-746.

Van Lange, P. A. M. \& Visser, K. (1999). Locomotion in social dilemmas: How people adapt to cooperative, tit-for-tat, and noncooperative partners. Journal of Personality and Social Psychology, 77, 762-773.

Wyer, R. S. (1969). Prediction of behavior in 2-person games. Journal of Personality and Social Psychology, 13), 222-238.

Zeelenberg, M., Nelissen, R. M. A., Breugelmans, S. M. \& Pieters, R. (2008). On emotion specificity in decision making: Why feeling is for doing. Judgment and Decision Making, 3, 18-27.
Table 7: SVO item endpoints and subsequent slopes that define each of the SVO Slider Measure items.

\begin{tabular}{ccccccc}
\hline & \multicolumn{2}{c}{ Endpoint 1} & \multicolumn{2}{c}{ Endpoint 2} & \multicolumn{2}{c}{ Descriptive information } \\
Item & Self & Other & Self & Other & Slope & Equation \\
\hline 1 & 85 & 85 & 85 & 15 & Undefined & $y \in[15,85], x=85$ \\
2 & 85 & 15 & 100 & 50 & 2.33 & $y=\frac{7}{3} \cdot x-\frac{550}{3}$ \\
3 & 50 & 100 & 85 & 85 & -0.43 & $y=-\frac{3}{7} \cdot x+\frac{850}{7}$ \\
4 & 50 & 100 & 85 & 15 & -2.43 & $y=-\frac{17}{7} \cdot x+\frac{1550}{7}$ \\
5 & 100 & 50 & 50 & 100 & -1.00 & $y=-1 \cdot x+150$ \\
6 & 100 & 50 & 85 & 85 & -2.33 & $y=-\frac{7}{3} \cdot x+\frac{850}{3}$ \\
\hline 7 & 100 & 50 & 70 & 100 & -1.67 & $y=-\frac{5}{3} \cdot x+\frac{650}{3}$ \\
8 & 90 & 100 & 100 & 90 & -1.00 & $y=-1 \cdot x+190$ \\
9 & 100 & 70 & 50 & 100 & -0.60 & $y=-\frac{3}{5} \cdot x+130$ \\
10 & 100 & 70 & 90 & 100 & -3.00 & $y=-3 \cdot x+370$ \\
11 & 70 & 100 & 100 & 70 & -1.00 & $y=-1 \cdot x+170$ \\
12 & 50 & 100 & 100 & 90 & -0.20 & $y=-\frac{1}{5} \cdot x+110$ \\
13 & 50 & 100 & 100 & 50 & -1.00 & $y=-1 \cdot x+150$ \\
14 & 100 & 90 & 70 & 100 & -0.33 & $y=-\frac{1}{3} \cdot x+\frac{370}{3}$ \\
15 & 90 & 100 & 100 & 50 & -5.00 & $y=-5 \cdot x+550$ \\
\hline
\end{tabular}

Note: The SVO Slider Measure has embedded in it several items which are also dictator games. Item number 5 from the primary set has this structure. From the secondary set, items 8, 11, 13 also have a slope of -1 , giving them the same tradeoff rate between the payoff for self and other but over different ranges. Results from these items can be analyzed separately. 
Figure 6: This shows the nine secondary SVO Slider items as seen by the subjects.

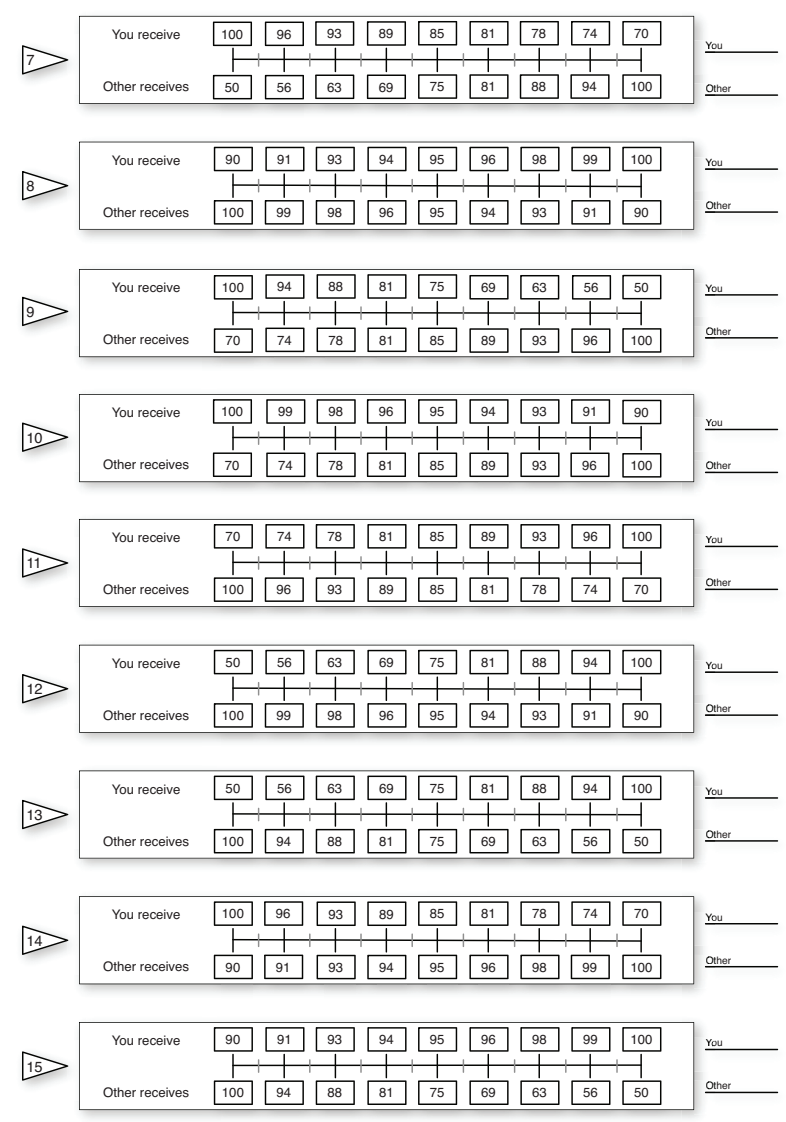

\section{Appendix}

\section{The items from the SVO Slider Measure SVO angle calculation}

1. Calculate the mean of the payoffs a subject allocated to herself across the six primary items $\left(\bar{A}_{s}\right)$.

2. Calculate the mean of the payoffs a subject allocated to the other person across the six primary items $\left(\bar{A}_{o}\right)$.

3. Subtract 50 from both means: $\bar{A}_{s}-50$ and $\bar{A}_{o}-50$.

4. In order to compute the SVO angle, calculate the inverse tangent of the ratio of the mean of the payoffs allocated to the other minus 50 and the mean of the payoffs allocated to the self minus 50:

$$
\mathrm{SVO}^{\circ}=\arctan \left(\frac{\left(\bar{A}_{o}-50\right)}{\left(\bar{A}_{s}-50\right)}\right)
$$

Figure 7: This figure shows the location of the nine secondary items of the Slider Measure in the self/other allocation plane.

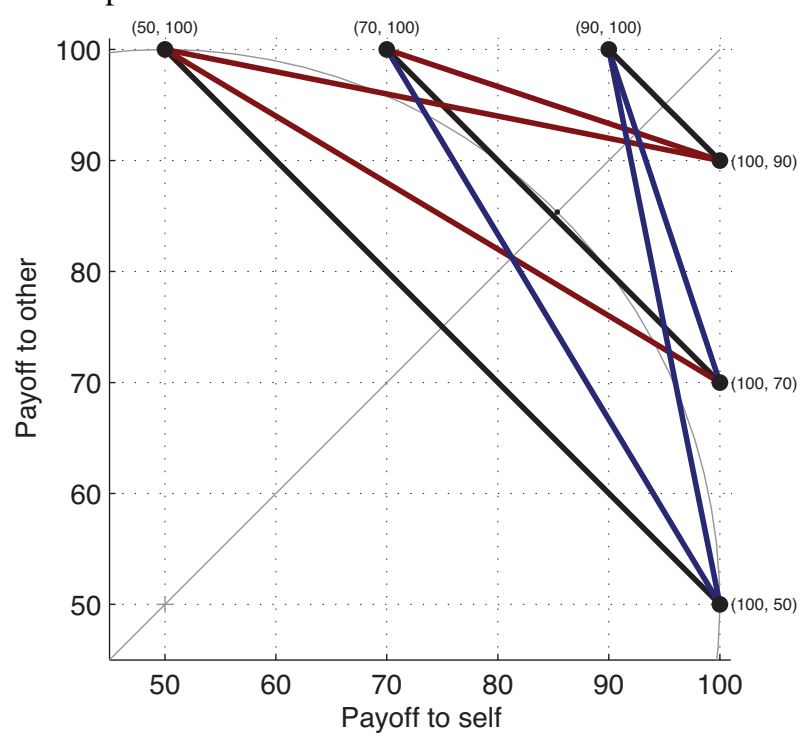

5. It is not recommended, but if for some reason categorical results are preferred to ratio level results, individual subjects' scores can be diminished to the categorical level following this scheme:

- Altruism: $\mathrm{SVO}^{\circ}>57.15^{\circ}$

- Prosociality: $22.45^{\circ}<\mathrm{SVO}^{\circ}<57.15^{\circ}$

- Individualism: $-12.04^{\circ}<\mathrm{SVO}^{\circ}<22.45^{\circ}$

- Competitiveness: $\mathrm{SVO}^{\circ}<-12.04^{\circ}$

\section{Explication of boundary determination}

The boundaries between categories were derived as follows:

If a subject would choose the option which maximizes the other one's payoff in each of the six primary items, the resulting angle would be $61.39^{\circ}$, indicating perfect altruism (see Table 8). Likewise, if a person would choose the option which maximizes the difference between the own and the other one's payoff in each of the six primary items, the resulting angle would be $-16.26^{\circ}$, indicating perfect competitiveness (see Table 11). For prosocial subjects, there are two ways in which they could answer the six primary items perfectly consistent (see Table 9). First, if a subject would choose the option which minimizes the difference between payoffs in each of the six items, the resulting angle would be $37.48^{\circ}$. Second, if a subject would choose the option which maximizes joint gain in each of the six items, the resulting angle would be between $37.09^{\circ}$ and $52.91^{\circ}$. The reason for this range is that this DM would be wholly indifferent across the entire SVO Slider item that has a slope of -1 (i.e., the item 
Table 8: Derivation of the SVO angle that would result if a person would consistently choose the altruistic options

\begin{tabular}{cccccccc}
\hline & \multicolumn{3}{c}{ Endpoint 1 } & \multicolumn{3}{c}{ Endpoint 2 } & \multicolumn{2}{c}{ Altruistic Choice } \\
Item & Self & Other & Self & Other & Self & Other \\
\hline 1 & 85 & 85 & 85 & 15 & 85 & 85 \\
2 & 85 & 15 & 100 & 50 & 100 & 50 \\
3 & 50 & 100 & 85 & 85 & 50 & 100 \\
4 & 50 & 100 & 85 & 15 & 50 & 100 \\
5 & 100 & 50 & 50 & 100 & 50 & 100 \\
6 & 100 & 50 & 85 & 85 & 85 & 85 \\
\hline Resulting means: & & & & 70 & 86.7 \\
Resulting means - 50: & & & & 20 & 36.7 \\
\hline Resulting angle: & & & \multicolumn{3}{c}{$61.39^{\circ}$} \\
\hline
\end{tabular}

with endpoints 100, 50 and 50, 100) as it has a constant sum. For the domain of individualism, if a subject would consistently choose the option which maximizes the own payoff in each of the six items, this would yield and angle between $-7.82^{\circ}$ and $7.82^{\circ}$ (see Table 10). The reason for this range is that this particular DM would be wholly indifferent across the entire SVO Slider item that has an undefined slope (endpoints 85, 85 and 85, 15).

The boundaries according to which subjects can be categorized were derived by bisecting the ranges between the angles that are produced when a subject with one of the four classical motivational orientations answers the Slider Measure perfectly consistent. When there is a range of angles which can be produced by perfectly consistent choice behavior (as is the case for individualistic and prosocial subjects), the maximum/minimum values are used for computing the boundaries. Concretely, the boundaries were calculated as follows:

- Boundary between altruism and prosociality:

$$
\frac{61.39^{\circ}+52.91^{\circ}}{2}=57.15^{\circ}
$$

- Boundary between prosociality and individualism:

$$
\frac{37.09^{\circ}+7.82^{\circ}}{2}=22.45^{\circ}
$$

- Boundary between individualism and competitiveness:

$$
\frac{-7.82^{\circ}+-16.26^{\circ}}{2}=-12.04^{\circ}
$$

Table 9: Derivation of the SVO angle that would result if a person would consistently choose the prosocial options

\begin{tabular}{ccccccc}
\hline & \multicolumn{2}{c}{ Endpoint 1 } & \multicolumn{2}{c}{ Endpoint 2 } & \multicolumn{2}{c}{ Prosocial Choice } \\
Item & Self & Other & Self & Other & Self & Other \\
\hline 1 & 85 & 85 & 85 & 15 & 85 & 85 \\
2 & 85 & 15 & 100 & 50 & 100 & 50 \\
3 & 50 & 100 & 85 & 85 & 85 & 85 \\
4 & 50 & 100 & 85 & 15 & 50 & 100 \\
5 & 100 & 50 & 50 & 100 & $100 \leftrightarrow 50$ & $50 \leftrightarrow 100$ \\
6 & 100 & 50 & 85 & 85 & 85 & 85 \\
\hline Resulting means: & & & & $84.2 \leftrightarrow 75.8$ & $75.8 \leftrightarrow 84.2$ \\
Resulting means $-50:$ & & & & $34.2 \leftrightarrow 25.8$ & $25.8 \leftrightarrow 34.2$ \\
\hline Resulting angle: & & & \multicolumn{3}{c}{$37.09^{\circ} \leftrightarrow 52.91^{\circ}$} \\
\hline
\end{tabular}

Table 10: Derivation of the SVO angle that would result if a person would consistently choose the individualistic options

\begin{tabular}{ccccccc}
\hline & \multicolumn{3}{c}{ Endpoint 1 } & \multicolumn{2}{c}{ Endpoint 2} & \multicolumn{2}{c}{ Individualistic Choice } \\
Item & Self & Other & Self & Other & Self & Other \\
\hline 1 & 85 & 85 & 85 & 15 & 85 & $85 \leftrightarrow 15$ \\
2 & 85 & 15 & 100 & 50 & 100 & 50 \\
3 & 50 & 100 & 85 & 85 & 85 & 85 \\
4 & 50 & 100 & 85 & 15 & 85 & 15 \\
5 & 100 & 50 & 50 & 100 & 100 & 50 \\
6 & 100 & 50 & 85 & 85 & 100 & 50 \\
\hline Resulting means: & & & & 92.5 & $55.8 \leftrightarrow 44.2$ \\
Resulting means - 50: & & & & 42.5 & $5.8 \leftrightarrow-5.8$ \\
\hline Resulting angle: & & \multicolumn{5}{c}{$-7.82^{\circ} \leftrightarrow 7.82^{\circ}$} \\
\hline
\end{tabular}

Table 11: Derivation of the SVO angle that would result if a person would consistently choose the competitive options

\begin{tabular}{ccccccc}
\hline & \multicolumn{3}{c}{ Endpoint 1 } & \multicolumn{2}{c}{ Endpoint 2 } & \multicolumn{2}{c}{ Competitive Choice } \\
Item & Self & Other & Self & Other & Self & Other \\
\hline 1 & 85 & 85 & 85 & 15 & 85 & 15 \\
2 & 85 & 15 & 100 & 50 & 85 & 15 \\
3 & 50 & 100 & 85 & 85 & 85 & 85 \\
4 & 50 & 100 & 85 & 15 & 85 & 15 \\
5 & 100 & 50 & 50 & 100 & 100 & 50 \\
6 & 100 & 50 & 85 & 85 & 100 & 50 \\
\hline Resulting means: & & & & & 90 & 38.3 \\
Resulting means - 50: & & & & 40 & -11.7 \\
\hline Resulting angle: & & & & & $-16.26^{\circ}$ \\
\hline
\end{tabular}

